ORIGINAL ARTICLE

\section{Remote Ischemic Preconditioning and Outcomes of Cardiac Surgery}

\author{
D.J. Hausenloy, L. Candilio, R. Evans, C. Ariti, D.P. Jenkins, S. Kolvekar, R. Knight, \\ G. Kunst, C. Laing, J. Nicholas, J. Pepper, S. Robertson, M. Xenou, T. Clayton, \\ and D.M. Yellon, for the ERICCA Trial Investigators*
}

A BSTRACT

\section{BACKGROUND}

Whether remote ischemic preconditioning (transient ischemia and reperfusion of the arm) can improve clinical outcomes in patients undergoing coronary-artery bypass graft (CABG) surgery is not known. We investigated this question in a randomized trial.

\section{METHODS}

We conducted a multicenter, sham-controlled trial involving adults at increased surgical risk who were undergoing on-pump CABG (with or without valve surgery) with blood cardioplegia. After anesthesia induction and before surgical incision, patients were randomly assigned to remote ischemic preconditioning (four 5-minute inflations and deflations of a standard blood-pressure cuff on the upper arm) or sham conditioning (control group). Anesthetic management and perioperative care were not standardized. The combined primary end point was death from cardiovascular causes, nonfatal myocardial infarction, coronary revascularization, or stroke, assessed 12 months after randomization.

\section{RESULTS}

We enrolled a total of 1612 patients (811 in the control group and 801 in the ischemic-preconditioning group) at 30 cardiac surgery centers in the United Kingdom. There was no significant difference in the cumulative incidence of the primary end point at 12 months between the patients in the remote ischemic preconditioning group and those in the control group (212 patients [26.5\%] and 225 patients [27.7\%], respectively; hazard ratio with ischemic preconditioning, 0.95 ; $95 \%$ confidence interval, 0.79 to $1.15 ; \mathrm{P}=0.58$ ). Furthermore, there were no significant between-group differences in either adverse events or the secondary end points of perioperative myocardial injury (assessed on the basis of the area under the curve for the high-sensitivity assay of serum troponin $\mathrm{T}$ at 72 hours), inotrope score (calculated from the maximum dose of the individual inotropic agents administered in the first 3 days after surgery), acute kidney injury, duration of stay in the intensive care unit and hospital, distance on the 6-minute walk test, and quality of life.

CONCLUSIONS

Remote ischemic preconditioning did not improve clinical outcomes in patients undergoing elective on-pump CABG with or without valve surgery. (Funded by the Efficacy and Mechanism Evaluation Program [a Medical Research Council and National Institute of Health Research partnership] and the British Heart Foundation; ERICCA ClinicalTrials.gov number, NCT01247545.)
The authors' full names, academic degrees, and affiliations are listed in the Appendix. Address reprint requests to Prof. Hausenloy at the Hatter Cardiovascular Institute, University College London, 67 Chenies Mews, London WCIE 6HX, United Kingdom, or at d.hausenloy@ucl.ac.uk.

*A complete list of investigators in the Effect of Remote Ischemic Preconditioning on Clinical Outcomes in Patients Undergoing Coronary Artery Bypass Graft Surgery (ERICCA) trial is provided in the Supplementary Appendix, available at NEJM.org.

Drs. Hausenloy and Candilio contributed equally to this article.

DOI: 10.1056/NEJMoa1413534 Copyright @ 2015 Massachusetts Medical Society. 
ORONARY HEART DISEASE IS THE LEADing cause of death and disability worldwide. For patients with multivessel coronary artery disease, the treatment of choice for many is revascularization by means of coronaryartery bypass grafting (CABG) surgery. As a result of the aging of the population, an increased prevalence of coexisting conditions (e.g., diabetes, obesity, and hypertension), and a growing need for concomitant valve surgery, higher-risk patients are undergoing CABG surgery (with or without valve surgery); the clinical outcomes in such patients have been worse than the outcomes in patients without so many problems. ${ }^{1,2}$ Thus, novel cardioprotective interventions are indicated to improve clinical outcomes.

There is evidence that the heart and other organs can be protected against lethal acute ischemia-reperfusion injury by applying one or more cycles of brief, nonlethal ischemia and reperfusion to a remote organ or tissue, a procedure that has been termed remote ischemic preconditioning. ${ }^{3-6}$ The mechanisms underlying remote ischemic preconditioning are incompletely understood, although current concepts suggest that a bloodborne factor is produced in response to the remote ischemic preconditioning stimulus, which conveys the protective effect from the remote organ or tissue to the target organ.,6 Remote ischemic preconditioning can be performed noninvasively by simply inflating and deflating a standard blood-pressure cuff placed on the upper arm or thigh to induce transient ischemia and reperfusion, ${ }^{7}$ an intervention that has been shown to reduce the extent of perioperative myocardial injury in patients undergoing CABG surgery (with or without valve surgery). ${ }^{8-10}$ Whether remote ischemic preconditioning can improve clinical outcomes after cardiac surgery is not known. We conducted the Effect of Remote Ischemic Preconditioning on Clinical Outcomes in Patients Undergoing Coronary Artery Bypass Graft Surgery (ERICCA) trial to investigate this question. ${ }^{11}$

\section{METHODS}

TRIAL DESIGN

Our trial was a multicenter, randomized, shamcontrolled clinical study conducted at 30 cardiac surgery centers in the United Kingdom. The study was approved by the National Health Service Research Ethics Committee and was conducted in accordance with the principles of Good Clinical Practice under the oversight of University College London Hospital. All participants provided written informed consent. The London School of Hygiene and Tropical Medicine Clinical Trials Unit coordinated the trial. Details of the trial design have been reported previously. ${ }^{11}$ The study adhered to the protocol, which is available with the full text of this article at NEJM.org.

The authors designed the study and collected, analyzed, and interpreted the data. All the authors wrote or contributed to the writing of the manuscript, made the decision to submit it for publication, and vouch for the accuracy and completeness of the data and analysis. The funding sources had no role in the study.

\section{PARTICIPANTS}

Eligible patients were adults ( $\geq 18$ years of age) with an additive European System for Cardiac Operative Risk Evaluation (EuroSCORE) of 5 or higher (with higher scores indicating a greater risk of death; 0 indicates minimum risk and $\geq 6$ indicates high risk) who were undergoing onpump CABG (with or without valve surgery) with myocardial protection provided by blood cardioplegia. Exclusion criteria were cardiogenic shock or cardiac arrest during the current admission, pregnancy, clinically significant peripheral arterial disease affecting the arms, hepatic dysfunction (bilirubin level of $>20 \mu \mathrm{mol}$ per liter [1.2 mg per deciliter] or international normalized ratio of >2.0), pulmonary disease (forced expiratory volume in 1 second of $<40 \%$ of the predicted value) or renal failure (estimated glomerular filtration rate of $<30 \mathrm{ml}$ per minute per $1.73 \mathrm{~m}^{2}$ of body-surface area), and concomitant therapy with glibenclamide or nicorandil (medications that may interfere with remote ischemic preconditioning). Participants underwent follow-up assessments at 6 weeks (performed at the time of the routine outpatient clinic visit) and at 12 months (performed mainly by telephone contact with the patient or review of the general practitioner's notes, since there was no routine outpatient clinic visit at this time point).

\section{RANDOMIZATION}

Patients were randomly assigned in a 1:1 ratio to undergo either remote ischemic preconditioning or sham preconditioning (control group). Randomization was conducted by means of a secure 
website (Sealed Envelope) and was stratified according to recruiting center. The patients, anesthesiologists, cardiac surgeons, intensive care unit (ICU) and ward staff, and study investigators collecting and analyzing the data were all unaware of the treatment assignments.

\section{INTERVENTIONS}

For participants who were randomly assigned to undergo remote ischemic preconditioning, a standard blood-pressure cuff was placed on the upper arm, inflated to $200 \mathrm{~mm} \mathrm{Hg}$, and left inflated for 5 minutes. The cuff was then deflated to $0 \mathrm{~mm} \mathrm{Hg}$ and left uninflated for 5 minutes. This cycle was performed four times in total. If the systolic pressure was higher than $185 \mathrm{~mm} \mathrm{Hg}$, the cuff was inflated to $15 \mathrm{~mm} \mathrm{Hg}$ above the systolic pressure. The protocol for the control group comprised four 5-minute cycles of simulated remote ischemic preconditioning. Cuff inflation was simulated by inflating the cuff with the valve open; this prevented actual inflation of the cuff. After 5 minutes, the air valve was closed and left closed for 5 minutes, corresponding to cuff deflation. Remote ischemic preconditioning and sham preconditioning were performed after anesthesia induction and before surgical incision.

\section{SURGICAL PROCEDURE}

Anesthetic management and perioperative care were not standardized. Arterial blood pressure, central venous pressure, electrocardiographic tracings, and nasopharyngeal temperature were recorded continuously. Intravenous glyceryl trinitrate was used only when clinically indicated, since it may interfere with the cardioprotective effect of remote ischemic preconditioning. After the institution of standard nonpulsatile cardiopulmonary bypass, all coronary-artery bypass grafts were constructed while blood cardioplegia was being used for myocardial protection. After the anastomoses of the grafts (with or without valve replacement or repair), cardiopulmonary bypass was discontinued and protamine was used to reverse the effect of heparin.

\section{OUTCOMES}

The primary study end point was the rate of major adverse cardiac and cerebral events, assessed within 12 months after randomization. These comprised death from cardiovascular causes, nonfatal myocardial infarction, coronary revascularization, or stroke. All primary events were validated by an independent event validation committee. Definitions of the major clinical end points are provided in the Methods section in the Supplementary Appendix, available at NEJM.org.

Secondary end points included components of the primary end point, assessed at 30 days and 12 months, and death from any cause, assessed at 12 months. Additional secondary end points were perioperative myocardial injury (assessed on the basis of the area under the curve [AUC] at 72 hours for the results of a high-sensitivity troponin $\mathrm{T}$ assay [normal troponin $\mathrm{T}$ range, 0 to $14 \mathrm{ng}$ per liter] in blood samples obtained preoperatively and $6,12,24,48$, and 72 hours after cardiac bypass); grade 1,2 , or 3 acute kidney injury within 72 hours after surgery (assessed on the basis of the increase in the serum creatinine level according to the International Kidney Disease: Improving Global Outcomes classification $^{12}$ ); serum creatinine level at 6 weeks and 12 months; 24-hour AUC for plasma neutrophil gelatinase-associated lipocalin (NGAL, calculated from blood samples taken preoperatively and at 6,12 , and 24 hours after cardiac bypass; normal range, 0 to 1000 ng per liter); maximum inotrope score in the 72-hour postoperative period (an assessment of hemodynamic stability calculated from the maximum dose of the individual inotropic agents administered in the first 3 days after surgery ${ }^{13}$ ); length of ICU stay and hospital stay; 6-minute walk test at baseline, 6 weeks, and 12 months; and health-related quality of life as assessed by the European Quality of Life-5 Dimensions score at baseline, 6 weeks, and 3, 6, 9, and 12 months.

\section{STATISTICAL ANALYSIS}

On the basis of previous data, ${ }^{14,15}$ we estimated that $20 \%$ of participants in the control group would have major adverse cardiac or cerebral events within 12 months. To detect a $27 \%$ relative reduction in this primary end point in the ischemic-preconditioning group (from $20.0 \%$ to $14.6 \%$ ), with a power of $80 \%$ and a significance level of $5 \%$, we calculated that a sample of 770 patients would be required for each study group, or 1540 in total. To allow for dropouts (4.5\%) this total was increased to 1610 patients (805 patients in each group). 
The primary analysis compared the cumulative incidence of major adverse cardiac or cerebral events at 12 months between the ischemicpreconditioning and control groups, with the use of Cox proportional-hazards models and with censoring of data at the date of the adverse event, loss to follow-up, or withdrawal from the study or at 12 months. The difference in treatment effect between subgroups was examined by including an interaction between the treatment group and the subgroup variable. The primary analysis was conducted on an intention-totreat basis and included all participants. We also carried out a per-protocol analysis, which was restricted to participants who underwent remote ischemic preconditioning or sham preconditioning as specified by the protocol and underwent CABG surgery (with or without valve surgery).

To compare subgroups with respect to the effect of treatment on the incidence of major adverse cardiac and cerebral events, we included an interaction between treatment group and the subgroup variable in the time-to-event model. Time-to-event methods were used to evaluate major adverse cardiac and cerebral events at 30 days, components of the primary end point at 30 days and 12 months, and deaths from any cause at 12 months. Simple linear regression was used for between-group comparisons of the natural logarithm of the AUC for troponin $\mathrm{T}$ at 72 hours and the AUC for NGAL at 24 hours. Nonparametric tests were used to compare the ischemicpreconditioning and control groups with respect to acute kidney injury and inotrope score. Proportional-hazards models accounting for the competing risk of death were used for hospital stay and ICU stay. Analysis of covariance, with adjustment for baseline values, was performed to evaluate the quality of life at baseline, at 6 weeks, and at $3,6,9$, and 12 months and the serum creatinine level at 6 weeks and 12 months. For quality of life, we used nonparametric confidence intervals because of the nonnormal distribution. A linear mixed model with unstructured residuals was used to compare the mean distance on the 6-minute walk test between the two study groups at 6 weeks and 12 months. Logistic regression was performed for between-group comparisons of the proportion of participants with postoperative atrial fibrillation, which was a post hoc outcome. Initial models included only participants for whom all data were available (complete-case analysis). For the complete-case analysis of the high-sensitivity assay for troponin T and of NGAL, data had to be available for all time points; for the complete-case analysis of serum creatinine, data had to be available at baseline and at the relevant follow-up visit. We evaluated the effect of missing data by using multiple imputation to replace any missing values for high-sensitivity troponin T assays, NGAL, and serum creatinine. Full details of the statistical methods, including the imputation model, are provided in the Methods section in the Supplementary Appendix.

\section{RESULTS}

\section{STUDY POPULATION}

Between April 2011 and March 2014, we enrolled 1612 patients undergoing on-pump CABG (with or without valve surgery) with blood cardioplegia (811 assigned to sham conditioning and 801 to remote ischemic preconditioning) (Fig. S1 in the Supplementary Appendix). Detailed screening logs of 1 month's duration were obtained for 3 separate, randomly selected months during the 36-month recruitment period. Of the 1869 patients who were screened during the 3 months, $414(22.2 \%)$ were eligible for inclusion in the study, and 195 of the eligible patients (47.1\%) were randomly assigned to a study group. A EuroSCORE of less than 5 was the main reason for ineligibility.

The treatment groups were well balanced with respect to baseline characteristics, surgical details, and use of cardiovascular medications during follow-up (Table 1, and Tables S1, S2, and S3 in the Supplementary Appendix). The intervention was completed according to the protocol for 716 (88.3\%) of the participants in the control group and 691 (86.3\%) of those in the ischemicpreconditioning group; reasons for incomplete intervention are provided in Figure S1 in the Supplementary Appendix. The mean $( \pm S D)$ time between the start of preconditioning and initiation of cardiac bypass was $1.8 \pm 0.6$ hours in the control group and $1.7 \pm 0.7$ hours in the ischemicpreconditioning group. Relatively few participants were lost to follow-up before 12 months for reasons other than death: 28 (3.5\%) in the control group and 19 (2.4\%) in the ischemicpreconditioning group (Fig. S1 in the Supplementary Appendix). All 1612 patients were included in the analysis of the primary outcome. 


\section{OUTCOMES}

There was no significant difference between the ischemic-preconditioning group and the control group with respect to the primary end point (proportion of participants with a major adverse cardiac or cerebral event at 12 months, $26.5 \%$ [212 participants] and 27.7\% [225 participants], respectively; hazard ratio with ischemic preconditioning, 0.95; 95\% confidence interval [CI], 0.79 to $1.15 ; \mathrm{P}=0.58$ ) (Table 2 and Fig. 1 , and Table S4 in the Supplementary Appendix). There was no evidence of a significant difference between the control group and the ischemic-conditioning group in any of the individual components of the primary end point (death from cardiovascular causes, nonfatal myocardial infarction, stroke, or coronary revascularization) (Table 2, and Figs. S2, S3, and S4 and Table S5 in the Supplementary Appendix).

Results of the per-protocol analysis were very similar to those of the intention-to-treat analysis, showing little difference in the incidence of major adverse cardiac and cerebral events between the ischemic-preconditioning and control groups. In the per-protocol analysis, $28.5 \%$ of participants (204 of 717) in the ischemic-preconditioning group had major adverse cardiac and cerebral events within 12 months, as compared with $27.2 \%$ (188 of 691 ) in the control group (hazard ratio, $0.95 ; 95 \% \mathrm{CI}, 0.78$ to $1.16 ; \mathrm{P}=0.64$ ). There was no evidence that the effect of remote ischemic preconditioning as compared with the effect of sham preconditioning differed significantly within the subgroups for any of the prespecified end points (Fig. 2). Post hoc subgroup analyses showed no evidence that the effect of remote ischemic preconditioning on the primary composite end point differed significantly according to the type of anesthetic used during surgery or the interval between the start of remote ischemic preconditioning and initiation of cardiac bypass (Table S6 in the Supplementary Appendix).

\section{SECONDARY OUTCOMES}

The complete-case analysis, which included 728 patients with full data on perioperative myocardial injury (45.2\% of all 1612 patients), suggested a $10 \%$ lower AUC for the troponin $\mathrm{T}$ level in patients undergoing remote ischemic preconditioning as compared with those undergoing sham preconditioning (geometric mean, 32.7 vs.

\begin{tabular}{|c|c|c|}
\hline Characteristic & $\begin{array}{c}\text { Control } \\
(N=811)\end{array}$ & $\begin{array}{c}\text { RIPC } \\
(\mathrm{N}=801)\end{array}$ \\
\hline \multicolumn{3}{|l|}{ Patients } \\
\hline Male sex - no./total no. (\%) & $586 / 806(72.7)$ & $556 / 790(70.4)$ \\
\hline Age $-y r$ & $76.3 \pm 7.0$ & $76.1 \pm 6.1$ \\
\hline \multicolumn{3}{|l|}{ EuroSCORE† } \\
\hline Median & 6 & 6 \\
\hline Range & $5-16$ & $5-17$ \\
\hline \multicolumn{3}{|l|}{ Prior diagnoses - no./total no. (\%) } \\
\hline Diabetes mellitus & $211 / 807(26.1)$ & $203 / 790(25.7)$ \\
\hline Hypercholesterolemia & $555 / 807(68.8)$ & $570 / 790(72.2)$ \\
\hline Hypertension & $599 / 807(74.2)$ & $602 / 790(76.2)$ \\
\hline Myocardial infarction & $309 / 807(38.3)$ & $328 / 790(41.5)$ \\
\hline Sulfonylurea — no./total no. (\%) & $49 / 807(6.1)$ & $42 / 791(5.3)$ \\
\hline \multicolumn{3}{|l|}{ Surgery } \\
\hline CABG completed — no./total (\%) & $776 / 805(96.4)$ & $772 / 789(97.8)$ \\
\hline Valve surgery — no./total no. (\%) & $406 / 775(52.4)$ & $371 / 772(48.1)$ \\
\hline \multicolumn{3}{|l|}{ Cross-clamp time $-\min t$} \\
\hline Median & 71 & 69 \\
\hline Range & $15-292$ & $18-324$ \\
\hline \multicolumn{3}{|l|}{ Cardiopulmonary-bypass time $-\min \mathbb{S}$} \\
\hline Median & 107 & 105 \\
\hline Range & $29-422$ & $34-585$ \\
\hline \multicolumn{3}{|l|}{ Anesthetics - no./total no. (\%) } \\
\hline Volatile, without propofol & $11 / 767(1.4)$ & $12 / 769(1.6)$ \\
\hline Volatile, with propofol & $312 / 767(40.7)$ & $313 / 769(40.7)$ \\
\hline Propofol, without volatile & $397 / 767(51.8)$ & $409 / 769(53.2)$ \\
\hline Other - no propofol or volatile & $47 / 767(6.1)$ & $35 / 769(4.6)$ \\
\hline Intravenous nitrates - no./total no. (\%) & $230 / 775(29.7)$ & $233 / 772(30.2)$ \\
\hline
\end{tabular}

* Plus-minus values are means \pm SD. Additional patient and surgical characteristics are provided in Tables S1 and S2 in the Supplementary Appendix. CABG denotes coronary-artery bypass grafting, and RIPC remote ischemic preconditioning.

$\dagger$ In the European System for Cardiac Operative Risk Evaluation (EuroSCORE), higher scores indicate a greater risk of death; 0 indicates minimum risk and $\geq 6$ indicates high risk. Data were available for 806 participants in the control group and 790 in the RIPC group.

$\Varangle$ Data were available for 734 participants in the control group and 735 in the RIPC group.

$\int$ Data were available for 750 participants in the control group and 757 in the RIPC group.

$36.4 \mathrm{ng} \cdot$ hour per milliliter; ratio, $0.90 ; 95 \% \mathrm{CI}$, 0.81 to 0.99 ). However, this effect largely disappeared when multiple imputation analyses were undertaken (geometric mean, 34.2 vs. 34.8 ng hour per milliliter; ratio, 0.98 ; $95 \% \mathrm{CI}, 0.91$ to 1.06) (Table 2). This lack of effect was sup- 


\begin{tabular}{|c|c|c|c|c|}
\hline Outcome & $\begin{array}{l}\text { Control } \\
(N=811)\end{array}$ & $\begin{array}{c}\text { RIPC } \\
(\mathrm{N}=801)\end{array}$ & $\begin{array}{c}\text { Treatment Effect, } \\
\text { Control vs. RIPC } \\
(95 \% \mathrm{CI})\end{array}$ & P Value \\
\hline \multicolumn{5}{|l|}{ Primary end point } \\
\hline MACCE within 12 mo & $225(27.7)$ & $212(26.5)$ & $0.95(0.79-1.15)$ & 0.58 \\
\hline $\begin{array}{l}\text { Death from cardiovascular causes - } \\
\text { no. (\%) }\end{array}$ & $32(3.9)$ & $47(5.9)$ & $1.50(0.96-2.35)$ & 0.08 \\
\hline Myocardial infarction & $191(23.6)$ & $173(21.6)$ & $0.91(0.74-1.12)$ & 0.39 \\
\hline Stroke & $16(2.0)$ & $17(2.1)$ & $1.08(0.55-2.14)$ & 0.82 \\
\hline Coronary revascularization & $3(0.4)$ & $2(0.2)$ & $0.68(0.11-4.09)$ & 0.68 \\
\hline \multicolumn{5}{|l|}{ Secondary end points } \\
\hline \multicolumn{5}{|l|}{ AUC for troponin $\mathrm{T}-\mathrm{ng} \cdot \mathrm{hr} / \mathrm{ml}$} \\
\hline Median (IQR) & $35.7(22.8-57.3)$ & $30.1(20.3-53.9)$ & & \\
\hline Geometric mean & $36.4 \pm 2.0$ & $32.7 \pm 2.0$ & & \\
\hline No. with complete data set & 367 & 361 & $0.90(0.81-0.99)$ & 0.03 \\
\hline Total no. with multiple imputation & 798 & 782 & $0.98(0.91-1.06)$ & 0.63 \\
\hline \multicolumn{5}{|l|}{ Inotrope score $†$} \\
\hline Median (IQR) & $6(0-16)$ & $6(0-15)$ & NA & 0.92 \\
\hline No. with data available & 794 & 775 & & \\
\hline Acute kidney injury — no./total no. (\%) & $293 / 772(38.0)$ & $287 / 749(38.3)$ & NA & 0.98 \\
\hline Grade 1 & $226 / 772(29.3)$ & $230 / 749(30.7)$ & & \\
\hline Grade 2 & $44 / 772(5.7)$ & $38 / 749(5.1)$ & & \\
\hline Grade 3 & $23 / 772(3.0)$ & $19 / 749(2.5)$ & & \\
\hline \multicolumn{5}{|l|}{ Hospital stay — days } \\
\hline Median (IQR) & $10(7-17)$ & $10(7-16)$ & NA & 0.36 \\
\hline No. with data available & 775 & 758 & & \\
\hline \multicolumn{5}{|l|}{ ICU stay — days } \\
\hline Median (IQR) & $3(1-5)$ & $2(1-4)$ & NA & 0.35 \\
\hline No. with data available & 775 & 758 & & \\
\hline \multicolumn{5}{|l|}{ Distance on 6 -min walk test $-\mathrm{m}$} \\
\hline At 6 wk & $335 \pm 125$ & $332 \pm 109$ & $-4(-24$ to 7$)$ & 0.72 \\
\hline At $12 \mathrm{mo}$ & $365 \pm 128$ & $386 \pm 116$ & 23 (2 to 44$)$ & 0.03 \\
\hline No. with data available & 402 & 383 & & \\
\hline
\end{tabular}

* Plus-minus values are means \pm SD. The treatment effect was calculated as the hazard ratio for the primary end point and as the ratio of geometric means for all the secondary end points except the distance on the 6-minute walk test, for which the treatment effect was calculated as the difference in means. More detailed data and other outcomes are provided in Tables S4 and S5 in the Supplementary Appendix. AUC denotes area under the curve, $\mathrm{Cl}$ confidence interval, ICU intensive care unit, IQR interquartile range, MACCE major adverse cardiac and cerebral events, and NA not applicable.

$\dagger$ Inotrope score was calculated from the maximum dose of the individual inotropic agents administered during the first 72 hours after surgery. ${ }^{13}$ The scale ranges from 0 to no upper limit, with higher scores indicating greater hemodynamic instability.

$\ddagger$ Grade 1, 2, or 3 acute kidney injury within 72 hours after surgery was assessed on the basis of the increase in the serum creatinine level according to the International Kidney Disease: Improving Global Outcomes classification. ${ }^{12}$ 
ported by examination of the data for the 1282 patients $(79.5 \%)$ who had at least one perioperative high-sensitivity troponin $\mathrm{T}$ assay result, which showed little difference at any time point between the ischemic-preconditioning and control groups (full details on patterns of missing data are provided in Table S7 in the Supplementary Appendix). Post hoc subgroup analyses showed no evidence that the effect of remote ischemic preconditioning on perioperative myocardial injury differed according to the type of anesthetic used during surgery or the interval between the start of remote ischemic preconditioning and initiation of cardiac bypass (Table S6 in the Supplementary Appendix).

The distance walked on the 6-minute walk test at 12 months was greater for participants in the ischemic-preconditioning group than for those in the control group (Table 2), although this finding should be interpreted with caution, since only 785 participants completed one or more of the three protocol-specified 6-minute walk tests (at baseline, 6 weeks, or 12 months), and of these participants, only 360 completed the 6-minute walk test at 12 months. There was no evidence of any effect of remote ischemic preconditioning on any of the other secondary end points (Table 2, and Table S8 in the Supplementary Appendix).

\section{ADVERSE EVENTS}

The proportion of participants with adverse events was similar in the ischemic-preconditioning and control groups (364 of 801 participants [45.4\%] and 354 of 811 [43.6\%], respectively) (Table 3). More participants in the ischemicpreconditioning group than in the sham-conditioning group had skin petechiae at the time of the intervention (35 of 801 participants [4.4\%] vs. 2 of $811[0.2 \%]$ ); there were no long-term consequences in either group. Three unexpected serious adverse events occurred at the time of the study intervention. Only one of these events was thought to be related to the intervention the blood-pressure cuff used in the ischemicpreconditioning intervention remained inflated during surgery, but this had no long-term consequences. The other two events, arm twitching and arm weakness or altered sensation, were thought to be unrelated to the intervention. A

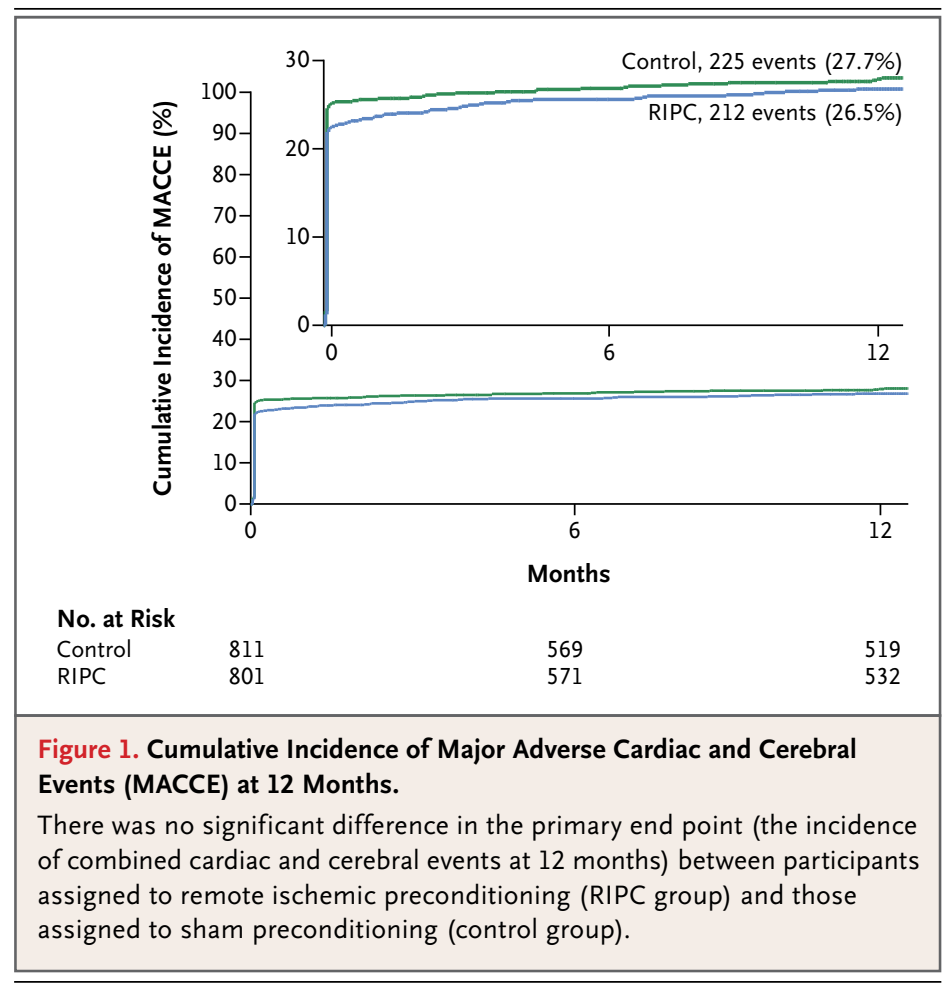

similar proportion of participants in the ischemic-preconditioning and sham-preconditioning groups had adverse events after the intervention (349 of the 801 participants assigned to remote ischemic preconditioning [43.6\%] and 353 of the 811 assigned to the sham conditioning [43.5\%]); none of these events were thought to be related to the intervention. Unexpected serious adverse events included heart failure, cardiac arrest, collapse or fall, diarrhea, noncardiac chest pain, shortness of breath, multiorgan failure, pulmonary edema, cancer (nonfatal), and confusion. Although there was a trend toward an increase in the rate of death from cardiovascular causes in association with remote ischemic preconditioning as compared with sham conditioning, the evidence for a difference was weak, and the study was not powered to detect this individual end point.

\section{DISCUSSION}

In higher-risk patients undergoing on-pump CABG (with or without valve surgery) with blood cardioplegia, remote ischemic preconditioning 


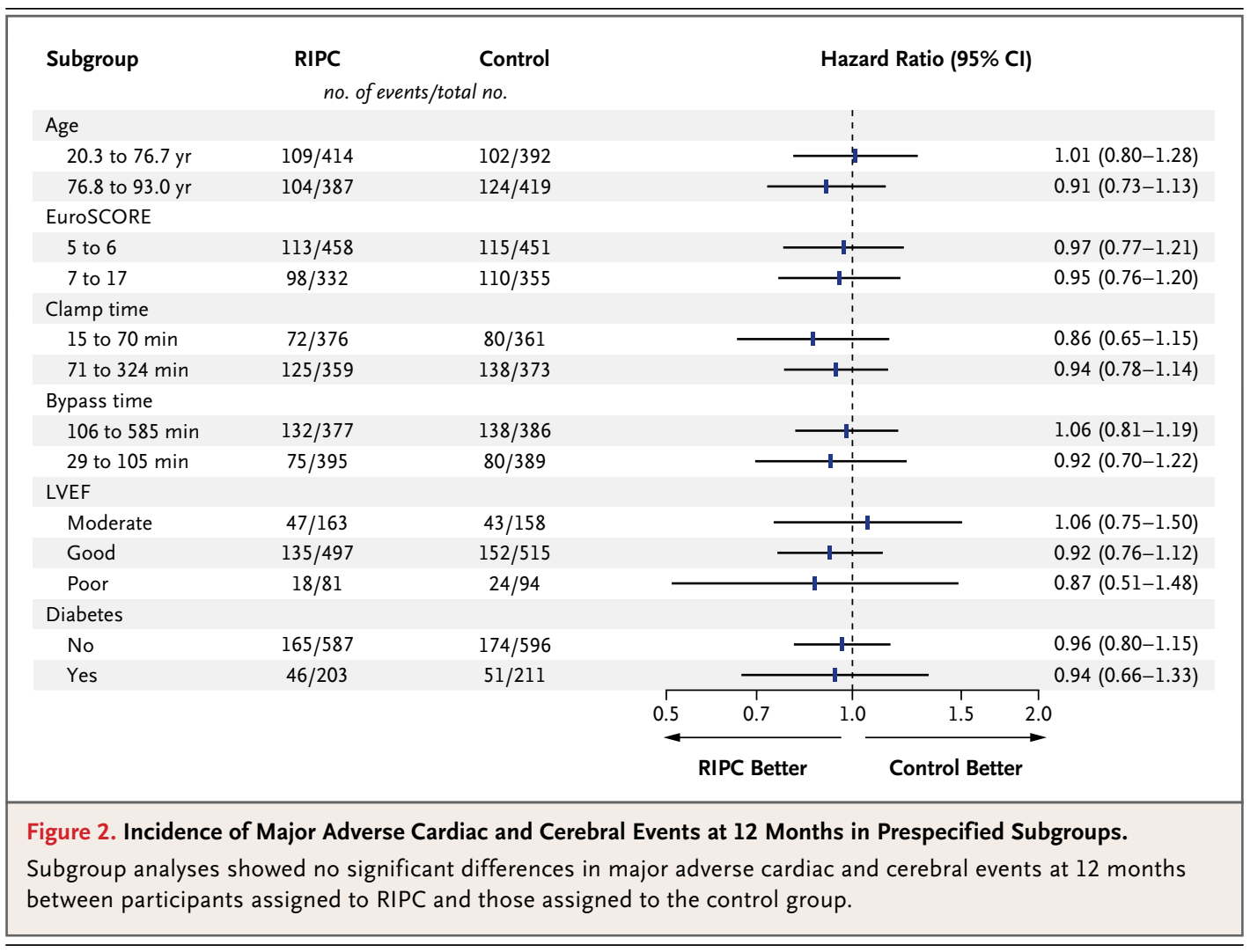

did not affect the incidence of major adverse cardiac and cerebral events at 12 months, as compared with sham preconditioning. Furthermore, remote ischemic preconditioning had no effect on any of the major secondary end points, as compared with sham preconditioning.

In two recent studies, analysis of secondary end points showed beneficial effects of remote ischemic conditioning on short-term clinical outcomes $^{16}$ and long-term clinical outcomes ${ }^{17}$ after cardiac surgery, although the studies were not adequately powered to assess these end points. Recent clinical trials that were prospectively designed and adequately powered failed to show any beneficial effect of remote ischemic preconditioning on inpatient clinical outcomes among 299 children (primary end point, the duration of the postoperative hospital stay $)^{18}$ and 1280 adults (combined primary end point, death, myocardial infarction, arrhythmia, stroke, coma, renal failure, respiratory failure, cardiogenic shock, gastrointestinal complication, or multiorgan failure). ${ }^{19}$

Studies assessing the effect of remote ischemic preconditioning on perioperative myocardi- al injury (measured on the basis of serum cardiac biomarker release), a surrogate end point for cardioprotection that has been associated with worse clinical outcomes after surgery, have had mixed results.-10,16,20,21 In our trial, we were unable to show a clinically significant reduction in perioperative myocardial injury, although this result should be interpreted with caution because of missing data.

At some point during surgery, almost $90 \%$ of the patients in our trial received propofol, an anesthetic agent that has been reported to attenuate the cardioprotective efficacy of remote ischemic preconditioning during cardiac surgery. ${ }^{22-24}$ Use of propofol in most study participants appears to be a common denominator in many of the studies that have shown no significant cardioprotective benefit of remote ischemic preconditioning. ${ }^{25}$

In our study, there was a suggestion of an increased risk of death from cardiovascular causes in association with remote ischemic preconditioning as compared with the sham preconditioning. However, our study was not powered to detect a difference in this individual end point, 
and the finding should therefore be interpreted with caution. In a post hoc subgroup analysis involving patients undergoing off-pump cardiac surgery, Hong et al. ${ }^{19}$ found that remote ischemic preconditioning combined with postconditioning was associated with a significant increase in the composite outcome of inpatient major adverse events. In a 58-patient study by Lucchinetti et al., ${ }^{26}$ remote ischemic preconditioning was associated with an increase in the secondary perioperative composite end point of myocardial infarction and new arrhythmias, as compared with the control group, although there was no significant between-group difference in cardiovascular outcomes at 6 months. Furthermore, remote ischemic preconditioning was also associated with increased expression of gene sets related to tumor-necrosis-factor signaling, stem-cell and progenitor-cell activity, and hypertrophy. However, the overwhelming majority of previously published studies ${ }^{17,18}$ and meta-analy$\operatorname{ses}^{27-30}$ have not shown any harmful effects of remote ischemic preconditioning during cardiac bypass surgery, and in fact, one recent study showed a reduction in all-cause mortality with remote ischemic preconditioning. ${ }^{17}$

Limitations of the current study include the lack of standardization of preoperative and perioperative anesthesia and medication, since we wanted to reflect the current clinical practice of cardiac surgery as much as possible, and incomplete data for some secondary end points. In addition, the anesthesiologists may not have been completely unaware of the study-group assignments. Since we did not collect data on fluid management (blood transfusion and administration of crystalloids and colloids), the inotrope data should be interpreted with caution. To investigate further the effect of the missing data on the results, we used multiple imputation. For unbiased results, this approach requires the as-

\begin{tabular}{|c|c|c|}
\hline Event & $\begin{array}{l}\text { Control } \\
(N=811)\end{array}$ & $\begin{array}{c}\text { RIPC } \\
(\mathrm{N}=801)\end{array}$ \\
\hline & \multicolumn{2}{|c|}{ no. of participants (\%) } \\
\hline Any adverse event & $354(43.6)$ & $364(45.4)$ \\
\hline $\begin{array}{l}\text { Adverse event during RIPC or sham } \\
\text { preconditioning }\end{array}$ & $3(0.4)$ & $37(4.6)$ \\
\hline Unexpected adverse event & $1(0.1)$ & $2(0.2)$ \\
\hline Skin petechiae & $2(0.2)$ & $35(4.4)$ \\
\hline Adverse event after intervention & $353(43.5)$ & $349(43.6)$ \\
\hline Death & $55(6.8)$ & $71(8.9)$ \\
\hline Hospital admission & $267(32.9)$ & $257(32.1)$ \\
\hline Other & 88 (10.9) & $80(10.0)$ \\
\hline
\end{tabular}

sumption that data are missing completely at random given the variables included in the imputation model, rather than the more stringent assumption that data are missing completely at random, which is required for unbiased results with complete-case analysis. Finally, an echocardiographic substudy had been planned, but because of logistical issues, very few patients were included in the substudy; thus, no meaningful data on left ventricular function were available for analysis.

In conclusion, our study shows that in higherrisk patients undergoing on-pump CABG (with or without valve surgery) with blood cardioplegia, the addition of remote ischemic preconditioning (with transient arm ischemia and reperfusion) did not provide greater cardioprotection than sham control and, accordingly, did not improve clinical outcomes at 12 months.

\footnotetext{
Supported by grants from the Efficacy and Mechanism Evaluation Program (a Medical Research Council and National Institute of Health Research partnership) (09/100/05) and the British Heart Foundation.

Disclosure forms provided by the authors are available with the full text of this article at NEJM.org.
}

\section{APPENDIX}

The authors' full names and academic degrees are as follows: Derek J. Hausenloy, M.B., Ch.B., Ph.D., Luciano Candilio, M.D.(Res.), Richard Evans, B.A., Cono Ariti, M.Sc., David P. Jenkins, M.B., B.S., Shyam Kolvekar, M.B., B.S., M.Ch., Rosemary Knight, Dip.N., Gudrun Kunst, M.D., Ph.D., Christopher Laing, M.B., Ch.B., M.D.(Res.), Jennifer Nicholas, Ph.D., John Pepper, M.Chir., Steven Robertson, B.A., Maria Xenou, M.Sc., Tim Clayton, M.Sc., and Derek M. Yellon, Ph.D., D.Sc., for the ERICCA Trial Investigators

The authors' affiliations are as follows: the Hatter Cardiovascular Institute, University College London (D.J.H., L.C., M.X., D.M.Y.), the National Institute of Health Research University College London Hospitals Biomedical Research Centre (D.J.H., D.M.Y.), the Clinical Trials Unit, London School of Hygiene and Tropical Medicine (R.E., R.K., J.N., S.R., T.C.), the Nuffield Trust (C.A.), the Heart Hospital, University College London Hospital (S.K.); King's College London and King's College Hospital (G.K.); Royal Free Hospital (C.L.); and the National Institute of Health Research Cardiovascular Biomedical Research Unit at Royal Brompton and Harefield NHS Trust (J.P.), London, and Papworth Hospital, Cambridge (D.P.J.) — all in the United Kingdom; and the National Heart Research Institute Singapore, National Heart Centre Singapore (D.J.H.), and the Cardiovascular and Metabolic Disorders Program, Duke-National University of Singapore (D.J.H.) — both in Singapore. 
REFERENCES

1. Ferguson TB Jr, Hammill BG, Peterson ED, DeLong ER, Grover FL. A decade of change - risk profiles and outcomes for isolated coronary artery bypass grafting procedures, 1990-1999: a report from the STS National Database Committee and the Duke Clinical Research Institute. Ann Thorac Surg 2002;73:480-9.

2. Thorsteinsson K, Fonager K, Mérie C, et al. Age-dependent trends in postoperative mortality and preoperative comorbidity in isolated coronary artery bypass surgery: a nationwide study. EurJ Cardiothorac Surg 2015 February 19 (Epub ahead of print).

3. Przyklenk K, Bauer B, Ovize M, Kloner RA, Whittaker P. Regional ischemic 'preconditioning' protects remote virgin myocardium from subsequent sustained coronary occlusion. Circulation 1993;87:893-9. 4. Hausenloy DJ, Yellon DM. Remote ischaemic preconditioning: underlying mechanisms and clinical application. Cardiovasc Res 2008;79:377-86.

5. Sivaraman V, Pickard JM, Hausenloy DJ. Remote ischaemic conditioning: cardiac protection from afar. Anaesthesia 2015;70:732-48

6. Heusch G, B $\phi$ tker HE, Przyklenk K, Redington A, Yellon D. Remote ischemic conditioning. J Am Coll Cardiol 2015;65: 177-95.

7. Kharbanda RK, Mortensen UM, White $\mathrm{PA}$, et al. Transient limb ischemia induces remote ischemic preconditioning in vivo. Circulation 2002;106:2881-3.

8. Hausenloy DJ, Mwamure PK, Venugopal V, et al. Effect of remote ischaemic preconditioning on myocardial injury in patients undergoing coronary artery bypass graft surgery: a randomised controlled trial. Lancet 2007;370:575-9.

9. Venugopal V, Hausenloy DJ, Ludman A, et al. Remote ischaemic preconditioning reduces myocardial injury in patients undergoing cardiac surgery with coldblood cardioplegia: a randomised controlled trial. Heart 2009;95:1567-71.

10. Thielmann M, Kottenberg E, Boengler $\mathrm{K}$, et al. Remote ischemic preconditioning reduces myocardial injury after coronary artery bypass surgery with crystalloid cardioplegic arrest. Basic Res Cardiol 2010;105:657-64.

11. Hausenloy DJ, Candilio L, Laing C, et al. Effect of Remote Ischemic Preconditioning on Clinical Outcomes in Patients Undergoing Coronary Artery Bypass Graft
Surgery (ERICCA): rationale and study design of a multi-centre randomized double-blinded controlled clinical trial. Clin Res Cardiol 2012;101:339-48.

12. Kellum JA, Lameire N. Diagnosis evaluation, and management of acute kidney injury: a KDIGO summary. Crit Care 2013;17:204.

13. Ko WJ, Lin CY, Chen RJ, Wang SS, Lin FY, Chen YS. Extracorporeal membrane oxygenation support for adult postcardiotomy cardiogenic shock. Ann Thorac Surg 2002;73:538-45.

14. Lee MS, Kapoor N, Jamal F, et al. Comparison of coronary artery bypass surgery with percutaneous coronary intervention with drug-eluting stents for unprotected left main coronary artery disease. J Am Coll Cardiol 2006;47:864-70.

15. Serruys PW, Morice MC, Kappetein $\mathrm{AP}$, et al. Percutaneous coronary intervention versus coronary-artery bypass grafting for severe coronary artery disease. N Engl J Med 2009;360:961-72.

16. Candilio L, Malik A, Ariti C, et al. Effect of remote ischaemic preconditioning on clinical outcomes in patients undergoing cardiac bypass surgery: a randomised controlled clinical trial. Heart 2015;101 185-92.

17. Thielmann M, Kottenberg E, Kleinbongard $\mathrm{P}$, et al. Cardioprotective and prognostic effects of remote ischaemic preconditioning in patients undergoing coronary artery bypass surgery: a singlecentre randomised, double-blind, controlled trial. Lancet 2013;382:597-604.

18. McCrindle BW, Clarizia NA, Khaikin $\mathrm{S}$, et al. Remote ischemic preconditioning in children undergoing cardiac surgery with cardiopulmonary bypass: a singlecenter double-blinded randomized trial. J Am Heart Assoc 2014;3(4):e000964. 19. Hong DM, Lee EH, Kim HJ, et al. Does remote ischaemic preconditioning with postconditioning improve clinical outcomes of patients undergoing cardiac surgery? Remote Ischaemic Preconditioning with Postconditioning Outcome Trial. Eur Heart J 2014;35:176-83.

20. Rahman IA, Mascaro JG, Steeds RP, et al. Remote ischemic preconditioning in human coronary artery bypass surgery: from promise to disappointment? Circulation 2010;122:Suppl:S53-S59.

21. Karuppasamy P, Chaubey S, Dew T, et al. Remote intermittent ischemia before coronary artery bypass graft surgery: a strategy to reduce injury and inflammation? Basic Res Cardiol 2011;106:511-9.

22. Kottenberg E, Thielmann M, Bergmann L, et al. Protection by remote ischemic preconditioning during coronary artery bypass graft surgery with isoflurane but not propofol - a clinical trial. Acta Anaesthesiol Scand 2012;56:30-8.

23. Kottenberg E, Musiolik J, Thielmann M, Jakob H, Peters J, Heusch G. Interference of propofol with signal transducer and activator of transcription 5 activation and cardioprotection by remote ischemic preconditioning during coronary artery bypass grafting. J Thorac Cardiovasc Surg 2014;147:376-82.

24. Bautin AE, Galagudza MM, Datsenko SV, et al. Effects of remote ischemic preconditioning on perioperative period in elective aortic valve replacement. Anesteziol Reanimatol 2014;3:11-7. (In Russian.)

25. Heusch G. Cardioprotection: chances and challenges of its translation to the clinic. Lancet 2013;381:166-75.

26. Lucchinetti E, Bestmann L, Feng J, et al. Remote ischemic preconditioning applied during isoflurane inhalation provides no benefit to the myocardium of patients undergoing on-pump coronary artery bypass graft surgery: lack of synergy or evidence of antagonism in cardioprotection? Anesthesiology 2012;116:296310.

27. D'Ascenzo F, Cavallero E, Moretti C, et al. Remote ischaemic preconditioning in coronary artery bypass surgery: a metaanalysis. Heart 2012;98:1267-71.

28. Remote Preconditioning Trialists' Group. Remote preconditioning and major clinical complications following adult cardiovascular surgery: systematic review and meta-analysis. Int J Cardiol 2014;176: 20-31.

29. D'Ascenzo F, Moretti C, Omedè P, et al. Cardiac remote ischaemic preconditioning reduces periprocedural myocardial infarction for patients undergoing percutaneous coronary interventions: a metaanalysis of randomised clinical trials. EuroIntervention 2014;9:1463-71.

30. Zhang B, Zhou J, Li H, Zhou M, Chen A, Zhao Q. Remote ischemic preconditioning does not improve the clinical outcomes in patients undergoing coronary artery bypass grafting: a meta-analysis of randomized controlled trials. Int J Cardiol 2014;172(1):e36-e38.

Copyright (C) 2015 Massachusetts Medical Society. 